DOI:10.2478/rrlm-2019-0037

\title{
Acute effects of recreational soccer on inflammatory response and cardiac and skeletal muscle damage indicators
}

\author{
Alper Aslan ${ }^{1 *}$, Yasar Salc1 ${ }^{1}$, Bilal Bicer ${ }^{1}$, Nazan Savas ${ }^{2}$, Nizami Duran ${ }^{3}$ \\ 1.Hatay Mustafa Kemal University, School of Physical Education and Sport, \\ Department of Coaching Education, Turkey \\ 2. Hatay Mustafa Kemal University, Medical Faculty, Department of Public Health, Turkey \\ 3. Hatay Mustafa Kemal University, Medical Faculty, Department of Medical Microbiology, Turkey
}

\begin{abstract}
Introduction: Previous studies have indicated that acute bouts of strenuous, long duration exercise induce significant increases in the inflammatory profile and cardiovascular risk markers. Although recreational soccer (RS) is a widespread activity, there are no data on this topic. Thus, the aim of this study was to examine RS-induced changes in inflammatory, cardiac, and skeletal muscle damage indicators in young and middle-aged males. Methods: Twelve young and 11 middle-aged males participated in the study. The participants played 6v6 $1 \mathrm{~h} R S$, where heart rate (HR) responses and external loads (distance covered, number of accelerations/decelerations) were determined. Blood samples were taken immediately prior to and following the matches, and $2 \mathrm{~h}, 4 \mathrm{~h}, 24 \mathrm{~h}, 48$ $h$, and 72 later. Results: Absolute HR responses and the number of accelerations and decelerations were higher in young participants than the middle-aged participants $(p<0.05)$. RS increased high sensitivity cardiac troponin I (hs-cTnI) in almost all participants. A total of $83.3 \%$ of the young participants exceeded the upper reference limit (URL), whereas none of the middle-aged participants exceeded the URL. Hs-cTnI levels returned to baseline after 24 and $48 \mathrm{~h}$ in the middle-aged and young participants, respectively. High sensitivity C-reactive protein (hs-CRP) and creatine kinase $(C K)$ increased in the middle-aged participants $(p<0.05)$, and in the young participants, though not significantly $(p>0.05)$. RS also led to significant increases in lactate dehydrogenase (LDH) in both groups ( $p<0.05)$. Hs-CRP, CK, and LDH values returned to baseline levels within 48 to $72 \mathrm{~h}$, except for the LDH values of the young participants. Conclusion: $R S$ induced short-term increases in cardiac and skeletal muscle damage markers and the inflammatory profile in young and middle-aged RS participants.
\end{abstract}

Keywords: cardiovascular system, cardiac troponin, health and exercise, muscle damage, inflammation Received: $28^{\text {th }}$ May 2019; Accepted: 30 ${ }^{\text {th }}$ September 2019; Published: 10 ${ }^{\text {th }}$ October 2019

*Corresponding author: Alper Aslan, Mustafa Kemal University Hatay, Turkey

E-mail: alperaslan72@gmail.com 


\section{Introduction}

Previous studies have reported the positive effects of regular physical activity on several health parameters $(1-3)$; however, there remains uncertainty regarding the health benefits of acute bouts of exercise since strenuous and long-duration exercise induces significant increases in cardiovascular risk markers (4).

The release of cardiac biomarkers after endurance and ultra-endurance events has been well documented (5-9); however, reports on these exercise modalities have only documented the reasons for a significant cardiac troponin release without the presence of negative clinical symptoms (10). Many other studies have focused on troponin release following competitive soccer matches (11-13), reporting varying results of modest (11), nonsignificant (12), and significant (13) elevations in cardiac troponin levels during the post-match recovery period.

Intermittent exercise, such as soccer, involves forceful muscle contractions. In particular, concentric propulsive forces during the acceleration phase and eccentric braking forces during the deceleration phase are highly associated with skeletal muscle damage and inflammatory response $(14,15)$. Intermittent exercise-induced muscle damage markers increase immediately after exercise, typically peak between $24 \mathrm{~h}$ and $48 \mathrm{~h}$, and return to baseline values within $72-120 \mathrm{~h}$ (14-16).

Although many studies have examined these parameters in different soccer populations (13,15,17-19), no data exist for RS, a widespread activity for all populations. RS involves intermittent periods of high-intensity effort interspersed with low-intensity recovery periods. A large number of studies has demonstrated that the cardiovascular stress imposed on players exceeds $80 \%$ of the maximum heart rate $\left(\mathrm{HR}_{\max }\right)$ $(1,20)$. At least $20 \%$ of the total RS match time is spent at $>90 \%$ of $\mathrm{HR}_{\max }$, regardless of age, gender, or health status (20). Furthermore, players exert high-intensity effort that is expected to induce an inflammatory response and skeletal muscle damage.

Despite the popularity of RS, there are no existing data on whether RS activity increases cardiac-specific markers and the inflammatory response. Thus, the aim of the present study was to investigate RS-induced changes in inflammatory, cardiac, and skeletal muscle damage indicators in physically active, healthy young and middle-aged males.

\section{Materials and Methods}

\section{Subjects}

Twelve young and 11 middle-aged (one player was injured and therefore excluded from the study) healthy males voluntarily participated in the present study. The descriptive characteristics of the participants are illustrated in Table 1. The subjects had been participating in soccer matches for recreational purposes at least 3 months (3 to 5 months), $1 \mathrm{~h}$ per day and 2 days per week before the start of the study. None of the participants reported a personal or family history of cardiovascular disease or any other metabolic or systemic disorders. None of the participants had any acute medical conditions and had not taken any medications during 1 month prior to the study. Written informed consent was obtained from each subject after a detailed description of the aim and procedures of the study were provided. The Ethical Committee of the local university approved the experimental protocol.

\section{Exercise Training Protocol and Data Collection}

The participants played a $6 \mathrm{v} 6,1 \mathrm{~h}$ soccer match on a $30-50 \mathrm{~m}$ artificial grass pitch. During the matches, HR responses and activity profiles were recorded with a sampling frequency of 1 
Table 1. Descriptive values in addition to HR responses and activity profiles of participants during the recreational soccer match (Mean \pm Standard Deviation)

\begin{tabular}{lcc}
\hline & Young Males & Middle-Aged Males \\
\hline Age $($ years $)$ & $22.8 \pm 1.8^{\#}$ & $43.1 \pm 4.4$ \\
\hline Height $(\mathrm{cm})$ & $177.8 \pm 4.1$ & $174.6 \pm 6.3$ \\
\hline Body Weight $(\mathrm{kg})$ & $71.1 \pm 8.4^{\#}$ & $86.7 \pm 8.6$ \\
\hline BMI & $22.5 \pm 2.4^{\#}$ & $28.4 \pm 1.7$ \\
\hline Mean HR $(\mathrm{bpm})$ & $185.4 \pm 9.3^{*}$ & $168.0 \pm 11.6$ \\
\hline HRreserve $(\%)$ & $87.5 \pm 4.5$ & $84.7 \pm 5.3$ \\
\hline HRmax $(\mathrm{bpm})$ & $200.7 \pm 6.7^{*}$ & $4147 \pm 4.0$ \\
\hline Total Distance Covered $(\mathrm{m})$ & $4156 \pm 654.0$ & $3226.6 \pm 441.5$ \\
\hline LID $(0-11 \mathrm{~km} / \mathrm{h})$ & $3169.9 \pm 601.6$ & $347.5 \pm 164.7$ \\
\hline MID $(12-15 \mathrm{~km} / \mathrm{h})$ & $364.6 \pm 187.3$ & $89.6 \pm 43.1$ \\
\hline HID $(>15 \mathrm{~km} / \mathrm{s})$ & $161.1 \pm 127.9$ & $601 \pm 77.0$ \\
\hline Number of acceleration $\left(\mathrm{m} / \mathrm{sn}^{2}\right)$ & $709 \pm 52.8^{*}$ & $593 \pm 74.6$ \\
\hline Number of decelerations $\left(\mathrm{m} / \mathrm{sn}^{2}\right)$ & $694 \pm 39.4^{*}$ &
\end{tabular}

BMI: body mass index, Mean HR: mean heart rate during recreational soccer match, HRmax: maximal heart rate either during matches or age adjusted maximum (220-age), LID: distance covered in low intensity, MID: distance covered in moderate intensity, HID: distance covered in high intensity, " significantly lower than middle-aged males $(\mathrm{p}<0.05)$, * significantly higher than middle-aged males $(\mathrm{p}<0.05)$.

s and $10 \mathrm{~Hz}$ GPS units, respectively (Team Pro; Polar, Helsinki, Finland). The data were subsequently synchronized according to the manufacturer's guidelines and exported to an Excel worksheet, in which further analyses were performed. HR data were quantitated as the mean $\mathrm{HR}$ and the mean percentage of $\mathrm{HR}_{\text {reserve }}$ The percentage of $\mathrm{HR}_{\text {reserve }}$ was calculated using the following formula: $\% \mathrm{HR}_{\text {reserve }}=$ (match mean $\mathrm{HR}$ - resting $\mathrm{HR}) /\left(\mathrm{HR}_{\max }\right.$ - resting $\left.\mathrm{HR}\right) \times 100$. The highest HR value recorded during a match or the age-predicted maximal value $(220$ - age) was accepted as $\mathrm{HR}_{\max }$. The resting $\mathrm{HR}$ value corresponded to the minimal HR value from 10-min recordings in the supine position. Total distance covered, distance covered during low $(0-11 \mathrm{~km} / \mathrm{h})$, moderate $(11-15 \mathrm{~km} / \mathrm{h})$, and high $(>15 \mathrm{~km} / \mathrm{h})$ intensity running, and the number of decelerations and accelerations were also determined. The matches started at 12:00 pm, at a temperature and relative humidity of $20-22^{\circ} \mathrm{C}$ and $63-68 \%$, respectively (HI 8564; Hanna Instruments, Baranzate MI, Italy). The participants refrained from physical activity 5 days prior and until the end of the study.

\section{Blood Sampling and Analysis}

Blood samples $(7 \mathrm{~mL})$ were taken from the antecubital vein immediately prior to and following the matches, and $2 \mathrm{~h}, 4 \mathrm{~h}, 24 \mathrm{~h}, 48 \mathrm{~h}$, and 72 $\mathrm{h}$ later. The blood samples were centrifuged at 4,000 rpm for $5 \mathrm{~min}$, and the supernatant was transferred to two centrifuge tubes (one for biochemical parameter analysis and the other for an enzyme-linked immunosorbent assay) for each participant. Serum samples were stored at $-80^{\circ} \mathrm{C}$ until analysis. Biochemical analyses of the samples were performed in the central laboratory of the local University Medical Faculty Hospital. Troponin measurements were performed on a Siemens Advia Centaur XP instrument (Siemens, Latham, NY,USA) using kits compatible with this device, and tested according to the manufacturer's instructions. The URL for hs-cTnI was defined as the $99^{\text {th }}$ percentile of the healthy male participants, corresponding to $58 \mathrm{ng} / \mathrm{L}$ (21). High 
sensitivity C-reactive protein (hs-CRP) levels were measured using the BN system (Dade Behring, Newark, DE, USA). CK and LDH levels were measured using the Siemens Advia 1800 35 autoanalyzer (Siemens Healthcare Diagnostics Inc., Tarrytown, NY, USA) and compatible commercial kits (Advia Chemistry).

\section{Statistical Analysis}

All variables are expressed as the mean \pm standard deviation. The assumptions of homogeneity of variance and normality were tested using Hartley's $\mathrm{F}_{\text {max }}$ and Kolmogorov-Smirnov tests, respectively. The differences between the young and middle-aged participants in terms of descriptive characteristics, HR responses, and activity profiles were tested using an independent samples $t$-test. Repeated-measures analysis of variance (ANOVA) followed by the Bonferroni post-hoc test were performed to measure the differences between sampling times. The age differences with respect to baseline to post-exercise peak in the biomarkers were tested using an independent samples $t$-test. Pearson's correlation analyses were used to detect the relationships between the biomarkers and internal and external loading parameters during RS. Non-parametric versions of the test were performed when appropriate. All statistical analyses were performed using the SPSS for Windows, version 15.0 software (SPSS Inc., Chicago, IL, USA). A $p$-value $<0.05$ was considered statistically significant.

\section{Results}

The descriptive characteristics, HR responses, and activity profiles of the participants during $\mathrm{RS}$ are illustrated in Table 1.

Hs-cTnI levels were analyzed descriptively due to undetectable values at baseline and certain time points of the assessment. Hs-cTnI levels increased in all young participants, with $83.3 \%$
(10 players) exceeding the URL (Figure1A), whereas hs-cTnI increased in 10 out of the 11 middle-aged participants, but none exceeded the URL (Figure1B). Mean hs-cTnI levels in the young and middle-aged participants are shown
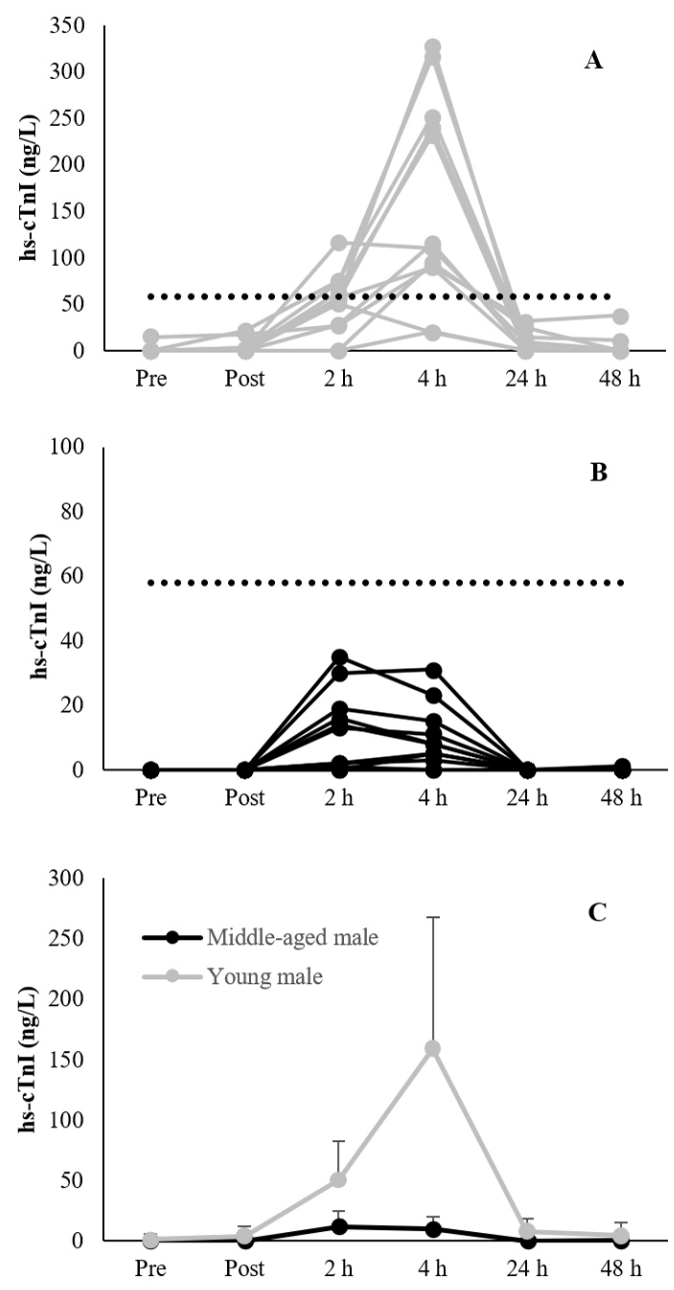

Fig. 1. A and B illustrate high sensitivity cardiac troponin I values for each young and middleaged male prior to and following the recreational soccer match, respectively. $C$ illustrates the mean high sensitivity cardiac troponin I values for young and middle-aged males. Horizontal line indicates the upper reference level $(58 \mathrm{mg} / \mathrm{L})$. 
in Figure 1C. None of the participants expressed any cardiovascular symptoms during the match or within the $48 \mathrm{~h}$ post-exercise period.

The results of repeated-measures ANOVA indicated significant differences between sampling times in hs-CRP levels only in middle-aged participants ( $p=0.000$, Figure 2). A post-hoc analysis indicated a significant increase in hs-CRP from baseline $(1.18 \pm 0.55 \mathrm{mg} / \mathrm{L})$ to $24 \mathrm{~h}$ postmatch $(2.42 \pm 1.06 \mathrm{mg} / \mathrm{L} ; p=0.001)$. The hs-CRP levels in the middle-aged participants tended to increase after the matches $(p>0.05)$. The baseline to peak increase in hs-CRP was similar between the age groups ( $p>0.05)$.

Repeated-measures ANOVA indicates significant differences between sampling times in $\mathrm{CK}$ levels in the young and middle-aged participants ( $p=0.001$ and $p=0.000$, respectively, Figure 3). However, even though CK values tented to increase in the young participants, Bonferroni posthoc analysis revealed that there were no significant differences between baseline CK levels and those at other time points $(p>0.05)$, whereas CK levels in the middle-aged participants increased from baseline $(122.09 \pm 49.08 \mathrm{U} / \mathrm{L})$ to immediately post-match $(167.36 \pm 66.07 \mathrm{U} / \mathrm{L} ; p=0.031)$ and $24 \mathrm{~h}$ later $(238.82 \pm 105.75 \mathrm{U} / \mathrm{L} ; p=0.025)$.

Significant differences in LDH were observed between sampling times in the young and middle-aged participants $(p=0.000$ and $p=0.004$, respectively, Figure 4). LDH levels in the young participants increased from baseline $(87.08 \pm 28.74 \mathrm{U} / \mathrm{L})$ to $24 \mathrm{~h}(184.42 \pm 32.46 \mathrm{U} / \mathrm{L}$; $p=0.000), 48$ h $(175.67 \pm 27.31 \mathrm{U} / \mathrm{L} ; p=0.000)$, and $72 \mathrm{~h}(165.92 \pm 29.19 \mathrm{U} / \mathrm{L} ; p=0.000)$ postmatch, whereas the LDH levels in the middle-aged participants only increased from baseline $(175.18 \pm 17.54 \mathrm{U} / \mathrm{L})$ to immediately postmatch $(212.45 \pm 26.46 \mathrm{U} / \mathrm{L} ; p=0.002)$. Baseline to peak LDH levels were significantly higher in the young than in the middle-aged participants $(p=0.000)$.

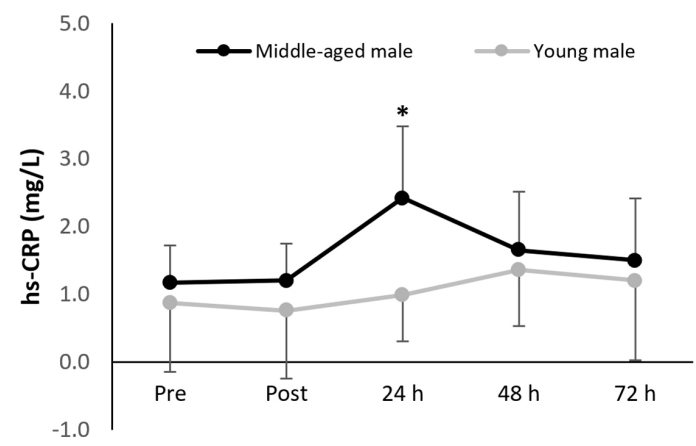

Fig. 2. High sensitivity $\mathrm{C}$-reactive protein levels prior to, immediately after, and $24 \mathrm{~h}, 48 \mathrm{~h}$, and $72 \mathrm{~h}$ following the recreational soccer match. *significantly higher than pre-match $(\mathbf{p}<0.05)$.

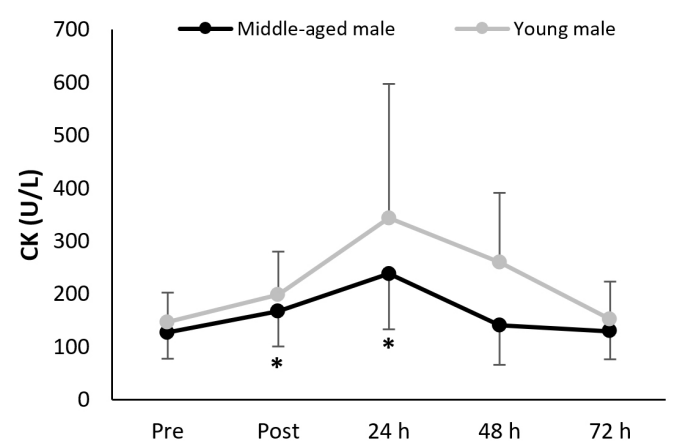

Fig. 3. Creatine kinase levels prior to, immediately after, and $24 \mathrm{~h}, 48 \mathrm{~h}$, and 72 $h$ following the recreational soccer match. *significantly higher than pre-match $(\mathbf{p}<0.05)$.

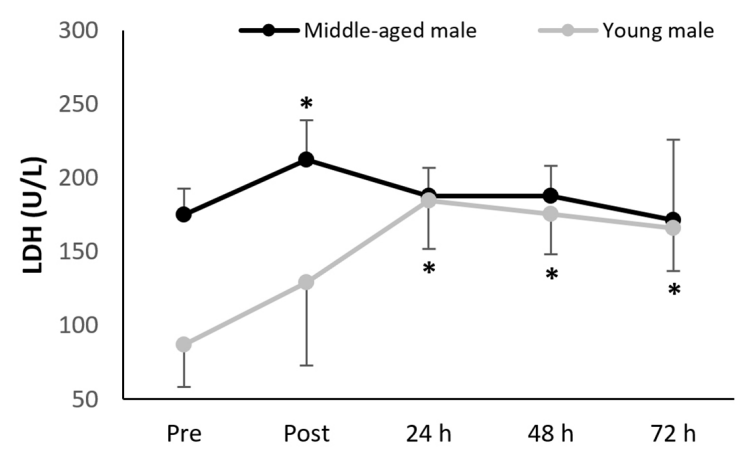

Fig. 4. Lactate dehydrogenase levels prior to, immediately after, and $24 \mathrm{~h}, 48 \mathrm{~h}$, and 72 $h$ following the recreational soccer match. *significantly higher than pre-match $(\mathbf{p}<0.05)$. 
The correlation between hs-cTnI and absolute HR was not significant $(p>0.05)$. In addition, no significant correlation was observed between hs-CRP, CK, or LDH, and the external load parameters (total distance covered, high-intensity distance, acceleration or deceleration; $p>0.05$ ).

\section{Discussion}

The purpose of the present study was to examine the effects of a $1 \mathrm{~h}$ RS match on biomarker responses in young and middle-aged males. This is the first study to examine age differences in cardiac and skeletal muscle damage, in addition to the inflammatory profile, in response to RS matches. The main findings indicate that RS caused substantial increases in hs-cTnI, hs-CRP, $\mathrm{CK}$, and LDH levels in young and middle-aged males, even though the time course of the increase and the sustainability in blood were different between the two groups.

Previous reports have indicated that the post-exercise increase in troponins is principally mediated by exercise intensity (22), which is generally evaluated by HR measurements and is also dependent on exercise duration when the intensity is fixed (22). In the present study, even though low-intensity distances constituted an important proportion of the total distance covered during $\mathrm{RS}$, the mean HR was approximately $85 \%$ of $\mathrm{HR}_{\text {reserve }}$ during the matches (Table 1). The high HR responses may occur in conjunction with energy-demanding activities, such as dribbling, tackling, shooting, and backward/sideways running, which contribute to the overall demand on players (23). In addition, HR can be affected by several other factors, such as increased catecholamines and hyperthermia, which can increase the mean values by causing the HR after high intensity activities to remain high even during subsequent low intensity activities. It has been well-established that increased core temperature and plasma catecholamines stimulate the sympa- thetic nervous system, leading to an increase in HR and force of contraction. Furthermore, a previous study observed that HR rarely decreases below $65 \%$ of $\mathrm{HR}_{\max }$ during a soccer match (23). These results demonstrate that, although the activity profile of soccer is intermittent in nature, players maintain an elevated cardiac output, HR, and systolic blood pressure throughout the activity. This sustained increase in cardiac work stresses the myocardium and could potentially damage cardiac cells (4).

$\mathrm{RS}$ resulted in increased hs-cTnI levels in almost all participants (with the exception of one middle-aged male). Moreover, 10 of the 12 young participants exceeded the URL at any of the time points of assessment, whereas none of the middle-aged participants reached such a high level (Figure 1A-B). Age also appears to be an important factor for the time kinetics of troponin release, since the baseline to peak increase and peak to baseline decrease took longer in young participants (Figure 1A-B). To the best of our knowledge, no study has investigated cardiac troponins in RS participants. However, although the findings of the present study are in accordance with the results obtained by Hosseini et al., (13) in adolescent soccer players and in studies conducted on other intermittent sports (24), the increase in troponin level was much higher in our young participants. The greater elevation in troponins may be explained by the higher absolute HR response (185.4 bpm) of our young players as compared with reference HR values observed during soccer matches (generally 165-175 bpm) (23). In addition, recent studies have indicated a significant negative correlation between post-exercise troponin levels and fitness level $(4,5)$; hence, potential differences in the fitness level of our subjects may also be a causative factor for the higher troponin levels seen in the present study. On the other hand, Carranza-García et al., (11), in a study of male and female soccer players, and Rahmana et al., (12), in elite soccer 
players, also showed only modest and nonsignificant increases in circulating post-exercise cTnI levels. These inconsistent observations between the present and aforementioned studies may also be attributed to differences in the fitness level of the participants and absolute HR responses, in addition to the type of troponin assay. Similar to the present study, recent studies using the latest highly sensitive methods have demonstrated that strenuous exercise leads to a significant elevation in cardiac-specific troponins in almost all participants (25).

The present results demonstrate that the external and relative internal loads $\left(\% \mathrm{HR}_{\text {reserve }}\right)$ imposed on the young and middle-aged participants were almost the same during RS; however, when the HR responses were expressed absolutely (bpm), the cardiac stress imposed on the young participants was higher than that imposed on the middle-aged participants (Table 1). Thus, the high absolute HR responses caused the cardiac stress to increase in the young participants, leading to higher post-match hs-cTnI levels. Similarly, Eijsvogels et al., (7) reported an inverse relationship between age and post-marathon cTnI levels, observing higher absolute HR responses during the race in younger athletes as compared with those in older athletes, which is in accordance with the present study. In addition, Knebel et al., (6) reported higher mean post-race cTnT levels in younger subjects following division of the athletes into younger $(<60$ years $)$ and older $(\geq 60$ years) age groups. Tian et al., (8) also observed higher post-exercise cTnT levels and a greater number of subjects exceeding the URL in adolescents than in adults following a 90-min treadmill run. In contrast, several studies performed in recreational and elite endurance athletes have shown that post-race troponin levels are unrelated to age $(9,26)$. Nevertheless, according to the current findings, young males exhibited a more pronounced cardiac troponin release after RS as compared with middle-aged males. Howev- er, using soccer simulation protocols is recommended to reveal biological age differences in troponin release after effort, since this method of data collection entirely eliminates the external load differences between participants.

Although several potential mechanisms have been suggested as causative factors for exercise-induced cardiac troponin release (25), the underlying mechanism has not yet been clearly defined. However, researchers have generally accepted the notion that physical exercise does not result in irreversible cardiac damage or necrosis. Several observations support this suggestion. Firstly, the time course of accumulation and clearance of troponins follows a different pattern after exercise than after acute myocardial infarction. Wu et al., (27) reported that serum troponin concentration increases within 4-12 h after the onset of acute myocardial infarction symptoms and remains abnormal for 4-10 days, whereas it peaks within 4-6 h after exercise and returns to the normal level within $24-48 \mathrm{~h}$ post-exercise, as seen in the present study. Furthermore, Nie et al., (28) examined the troponin responses following two consecutive exercise sessions performed with a recovery interval of $255 \mathrm{~min}$, and additional troponin was not observed after the second session. Moreover, a recent study using cardiac magnetic resonance imaging with late gadolinium enhancement also indicated that, even though cardiac troponins and $\mathrm{N}$-terminal pro-brain natriuretic peptide levels are high after endurance events, there is no evidence of any detectable myocardial damage or acute changes in left or right ventricular function (29). Additionally, similar to our participants, a previous study reported no unusual cardiovascular symptoms during or following exercise despite an increase in troponin levels (13). Finally, elite marathon runners have comparatively lower troponin levels than their non-elite counterparts after a race, which may occur in conjunction with improved resistance against exercise-induced cardiomyo- 
cyte membrane damage (5). Thus, elevated cardiac troponin levels may be linked to transient cardiomyocyte membrane damage, which acts as a physiological signal for cardiac muscle adaptation to exercise (4). Despite these observations, some studies have reported significant correlations between cardiac troponins and a decrease in post-exercise heart function $(30,31)$. Hence, in future studies, investigation of the underlying mechanism of troponin release and its acute and long-term effects on heart function is warranted. RS resulted in a marked increase in hs-CRP, which peaked 24-48 h post-match and returned to baseline within 2 days (Figure 2), as has been previously shown after a competitive soccer match $(14-15,17)$ and soccer simulation (19). On the other hand, the present study shows that baseline to peak increases in hs-CRP levels were higher in middle-aged than young participants. This finding may be due to the higher body mass index of the middle-aged participants (32). Previous studies have reported that exercise-induced increases in hs-CRP are linked to elevated interleukin (IL)-6, which is the main precursor for hepatic release of CRP (2). Initially, intramuscular signaling stimulates active muscles to release IL-6, and subsequently, exercise-induced muscle damage further stimulates IL-6 production (33). The release of IL-6 is mediated by exercise intensity, duration, and the amount of activated muscle mass (34). In fact, RS can be described as intermittent activity with frequent bursts of intense effort, which has the potential to cause muscle damage. Thus, the increase in hs-CRP in the present study may be linked to the increase in this myokine.

Accordingly, RS led to a transient increase in the inflammatory response, which may trigger acute cardiovascular risk factors in vulnerable individuals (2). However, Mendham et al., (3), in a similar group of participants (healthy middle-aged) and using a similar exercise regimen to the pres- ent study, indicated that a long-term small-sided rugby match improves the inflammatory profile by reducing cytokine production in muscle and adipose tissues, in addition to decreasing CRP concentrations. Nevertheless, future studies are needed to examine the reproducibility of this finding in diverse populations and to determine the threshold (intensity, volume, and weekly frequency) for intermittent exercise to enhance anti-inflammatory efficiency.

RS resulted in significant increases in CK and LDH levels, in accordance with other studies conducted in participants of recreational (35) and competitive (15-16,36) soccer matches. However, the blood kinetics of CK are somewhat higher and sustained in competitive players as compared with those in our recreational participants. Following competitive matches, CK immediately increases $(+70-250 \%)$, peaks $24-$ $48 \mathrm{~h}$ after the match, and returns to the normal level 48-120 h later, depending on the level of the peak; the greater the peak, the longer the time to return to normal (36). However, the present study indicates that CK increased immediately, peaked after $24 \mathrm{~h}$, and returned to baseline $48 \mathrm{~h}$ and $72 \mathrm{~h}$ later in middle-aged and young players, respectively. These fitness-related differences may be attributed to the larger muscle mass of competitive players, greater effort during high-intensity competitive matches, and longer competition times.

Bais and Edwards (37) indicated that acute responses of CK are positively correlated with the intensity and duration of exercise, and inversely correlated with conditioning level. When the same supramaximal exercise is performed, plasma CK levels are significantly lower in athletes as compared with those in matched sedanters (38). Furthermore, prior exercise sessions make skeletal muscles more resistant to further bouts of strenuous exercise, and any damage that does occur recovers at a faster rate (39). Thus, it can 
be speculated that the blood kinetics of CK would have been higher and more sustained in RS beginners as compared with the present findings, since our subjects had participated in RS for at least 3 months prior to data collection.

The present results show that RS resulted in a greater increase in LDH levels from baseline to peak in young as compared with middle-aged males. The CK rate of increase was also slightly, though not significantly, higher in young players. The insignificant difference may be due to the large variation in values. In addition, the recovery kinetics of these biomarkers after exercise took longer in the young males (Figures 3-4). A possible explanation for the higher and sustained increase in $\mathrm{CK}$ and $\mathrm{LDH}$ levels in young players may be linked to having a higher number of acceleration- and deceleration-type movements (Table 1), which has been previously indicated as a causative factor for mechanical muscle damage (37). In particular, the hamstring muscles contract eccentrically during deceleration-type movements to slow the forward movement of the leg. In fact, eccentric activation generates greater tension per cross-sectional area of contracting muscle mass as compared with concentric action, leading to greater disruption of structural proteins (39). On the other hand, the repair process of eccentric exercise-induced muscle damage is equally effective in physically active old and young individuals, with active older participants exhibiting almost the same capability to adapt to muscular damage as young participants (40). These results suggest that RS provides a sufficient stimulus for reconstructing skeletal muscle, leading to muscular development in a wide range of age groups. In fact, a previous study indicated the effectiveness of RS on muscular development in a diverse population when performed two or three times per week for 12 weeks (1).

In conclusion, although RS increased the acute inflammatory response, a previous study indi- cated that regular participation in intermittent exercise reduces the inflammatory profile (3). $\mathrm{RS}$ also resulted in muscle microtrauma, which could provide a stimulus for the improvement of muscular development. The results of the present study expand the current knowledge regarding increases in intermittent exercise-induced cardiac troponin levels. Furthermore, the young participants exhibited a more pronounced troponin increase than the middle-aged participants, which may be linked to their higher absolute HR responses. The increase in hs-cTnI returned to baseline within $24-48 \mathrm{~h}$ in middle-aged and young players. This transient increase was likely a result of exercise-induced myocardial membrane damage, which increased leakage of troponin from the cytosolic compartment. However, future studies are needed to reveal the underlying mechanism of troponin release following exercise and its clinical significance.

\author{
Abbreviations \\ CK: creatine kinase \\ HR: heart rate \\ hs-CRP: high sensitivity C-reactive protein \\ hs-cTnI: high sensitivity cardiac troponin I \\ LDH: lactate dehydrogenase \\ RS: recreational soccer \\ URL: upper reference limit
}

\section{Conflict of interest}

There are no conflicts of interest to report.

\section{Authors' contributions}

AA and YS: Conceptualization, methodology, formal analysis, investigation, original draft preparation, review and editing, visualization, project administration, and funding acquisition; $\mathrm{BB}, \mathrm{NS}$, and ND: formal analysis, investigation, review and editing, visualization, project administration, and funding acquisition. 


\section{Funding}

The present study was supported by the Research Fund of Mustafa Kemal University (No: 15742).

\section{References}

1. Krustrup P, Christensen JF, Randers MB, Pedersen $\mathrm{H}$, Sundstrup E, Jakobsen MD, et al. Muscle adaptations and performance enhancements of soccer training for untrained men. Eur J Appl Physiol. 2010 Apr 1;108(6):1247-58. DOI: 10.1007/s00421-009-1319-8

2. Futterman LG, Lemberg L. Regular physical exercise reduces cardiovascular risks. Am J Crit Care. 2006 Jan 1;15(1):99-102.

3. Mendham AE, Duffield R, Marino F, Coutts AJ. Small-sided games training reduces CRP, IL-6 and leptin in sedentary, middle-aged men. Eur J Appl Physiol. 2014 Nov 1;114(11):2289-97. DOI: 10.1007/ s00421-014-2953-3

4. Shave R, Baggish A, George K, Wood M, Scharhag J, Whyte $G$, et al. Exercise-induced cardiac troponin elevation: evidence, mechanisms, and implications. J Am Coll Cardiol. 2010 Jul 13;56(3):169-76. DOI: 10.1016/j.jacc.2010.03.037

5. Mehta R, Gaze D, Mohan S, Williams KL, Sprung $\mathrm{V}$, George K, et al. Post-exercise cardiac troponin release is related to exercise training history. Int J Sports Med. 2012 May;33(05):333-7. DOI: 10.1055/s-00311301322

6. Knebel F, Schimke I, Schroeckh S, Peters H, Eddicks S, Schattke S, et al. Myocardial function in older male amateur marathon runners: assessment by tissue Doppler echocardiography, speckle tracking, and cardiac biomarkers. J Am Soc Echocardiogr. 2009 Jul 1;22(7):8039. DOI: 10.1016/j.echo.2009.04.009

7. Eijsvogels TM, Hoogerwerf MD, Maessen MF, Seeger JP, George KP, Hopman MT, et al. Predictors of cardiac troponin release after a marathon. J Sci Med Sport. 2015 Jan 1;18(1):88-92. DOI: 10.1016/j.jsams.2013.12.002

8. Tian Y, Nie J, Huang C, George KP. The kinetics of highly sensitive cardiac troponin $\mathrm{T}$ release after prolonged treadmill exercise in adolescent and adult athletes. J Appl Physiol. 2012 May 31;113(3):418-25. DOI: 10.1152/japplphysiol.00247.2012

9. Neumayr G, Pfister R, Mitterbauer G, Maurer A, Gaenzer H, Sturm W, et al. Effect of the „Race Across The Alps" in elite cyclists on plasma cardiac troponins I and T. Am J Cardiol. 2002 Feb 15;89(4):484-6. DOI: 10.1016/S0002-9149(01)02280-9

10. George K, Whyte GP, Green DJ, Oxborough D, Shave RE, Gaze D, et al. The endurance athletes heart: acute stress and chronic adaptation. Br J Sports Med. 2012 Nov 1;46(Suppl 1):i29-36. DOI: 10.1136/ bjsports-2012-091141
11. Carranza-García LE, George K, Serrano-Ostáriz E, Casado-Arroyo R, Caballero-Navarro AL, Legaz-Arrese A. Cardiac biomarker response to intermittent exercise bouts. Int J Sports Med. 2011 May;32(05):32731. DOI: $10.1055 / \mathrm{s}-0030-1263138$

12. Rahnama N, Faramarzi M, Gaeini AA. Effects of intermittent exercise on cardiac troponin I and creatine kinase-MB. Int J Prev Med. 2011 Jan;2(1):20-3.

13. Hosseini SM, Azizi M, Samadi A, Talebi N, Gatterer H, Burtscher M. Impact of a soccer game on cardiac biomarkers in adolescent players. Pediatr Exerc Sci. 2018 Feb 1;30(1):90-5. DOI: 10.1123/pes.2017-0060

14. Ispirlidis I, Fatouros IG, Jamurtas AZ, Nikolaidis MG, Michailidis I, Douroudos I, et al. Time-course of changes in inflammatory and performance responses following a soccer game. Clin J Sports Med. 2008 Sep 1;18(5):42331. DOI: 10.1097/JSM.0b013e3181818e 0b

15. Souglis A, Bogdanis GC, Chryssanthopoulos C, Apostolidis N, Geladas ND. Time course of oxidative stress, inflammation, and muscle damage markers for 5 days after a soccer match: effects of sex and playing position. J Strength Cond Res. 2018 Jul 1;32(7):2045-54. DOI: $10.1519 /$ JSC.0000000000002436

16. Fatouros IG, Chatzinikolaou A, Douroudos II, Nikolaidis MG, Kyparos A, Margonis K, et al. Timecourse of changes in oxidative stress and antioxidant status responses following a soccer game. J Strength Cond Res. 2010 Dec 1;24(12):3278-86. DOI: 10.1519/ JSC.0b013e3181b60444

17. Souglis AG, Papapanagiotou A, Bogdanis GC, Travlos AK, Apostolidis NG, Geladas ND. Comparison of inflammatory responses to a soccer match between elite male and female players. J Strength Cond Res. 2015 May 1;29(5):1227-33. DOI: 10.1519/ JSC.0000000000000767

18. Souglis A, Travlos TK. The influence of competitive activity on selected biochemical and haematological parameters of amateur soccer athletes. J Phys Educ Sport. 2015 Mar 1;15(1):24-31.

19. Fransson D, Vigh-Larsen JF, Fatouros IG, Krustrup P, Mohr M. Fatigue responses in various muscle groups in well-trained competitive male players after a simulated soccer game. J Hum Kinet. 2018 Mar 23;61(1):85-97. DOI: 10.1515/hukin-2017-0129

20. Randers MB, Nybo L, Petersen J, Nielsen JJ, Christiansen L, Bendiksen M, Brito J, Bangsbo J, Krustrup P. Activity profile and physiological response to football training for untrained males and females, elderly and youngsters: influence of the number of players. Scand J Med Sci Sports, 2010 Apr;20(1):14-23. DOI: 10.1111/j.1600-0838.2010.01069.x

21. Collinson PO, Saenger AK, Apple FS. High sensitivity, contemporary and point-of-care cardiac troponin assays: educational aids developed by the IFCC Committee on Clinical Application of Cardiac Bio-Markers. 
Clin Chem Lab Med, 2019 May, 57(5):623-632. DOI: 10.1515/cclm-2018-1211

22. Fu F, Nie J, Tong TK. Serum cardiac troponin T in adolescent runners: effects of exercise intensity and duration. Int J Sports Med. 2009 Mar;30(03):168-72. DOI: $10.1055 / \mathrm{s}-0028-1104586$

23. Bangsbo J. The physiology of soccer--with special reference to intense intermittent exercise. Acta Physiol Scand Suppl. 1994;619:1-55.

24. Wedin JO, Henriksson AE. Postgame elevation of cardiac markers among elite floorball players. Scand J Med Sci Sports. 2015 Aug;25(4):495-500. DOI: 10.1111/sms. 12304

25. Gresslien T, Agewall S. Troponin and exercise. Int J Cardiol. 2016 Oct 15;221:609-21. DOI: 10.1016/j.ijcard.2016.06.243

26. Shave RE, Whyte GP, George K, Gaze DC, Collinson PO. Prolonged exercise should be considered alongside typical symptoms of acute myocardial infarction when evaluating increases in cardiac troponin T. Heart. 2005 Sep 1;91(9):1219-20. DOI: 10.1136/hrt.2004.046052

27. Wu AH, Apple FS, Gibler WB, Jesse RL, Warshaw MM, Valdes R. National Academy of Clinical Biochemistry Standards of Laboratory Practice: recommendations for the use of cardiac markers in coronary artery diseases. Clin Chem. 1999 Jul 1;45(7):1104-21.

28. Nie J, George KP, Tong TK, Tian Y, Shi Q. Effect of repeated endurance runs on cardiac biomarkers and function in adolescents. Med Sci Sports Exerc. 2011 Nov 1;43(11):2081-8. DOI: 10.1249/MSS.0b013e$31821 \mathrm{~d} 4 \mathrm{a} 82$

29. O'Hanlon R, Wilson M, Wage R, Smith G, Alpendurada FD, Wong J, et al. Troponin release following endurance exercise: is inflammation the cause? a cardiovascular magnetic resonance study. J Cardiovasc Magn Reson. 2010 Dec;12(1):38. DOI: 10.1186/1532-429X12-38

30. Tulloh L, Robinson D, Patel A, Ware A, Prendergast C, Sullivan D, Pressley L. Raised troponin T and echocardiographic abnormalities after prolonged strenuous exercise-the Australian Ironman Triathlon. Br J Sports Med. 2006 Jul 1;40(7):605-9. DOI: 10.1136/ bjsm.2005.022319

31. Shave R, Dawson E, Whyte G, George K, Gaze D, Collinson P. Altered cardiac function and minimal cardiac damage during prolonged exercise. Med Sci Sports Exerc. 2004 Jul 1;36(7):1098-103. DOI: 10.1249/01. MSS.0000131958.18154.1E

32. Wärnberg J, Cunningham K, Romeo J, Marcos A. Physical activity, exercise and low-grade systemic inflammation. Proc Nutr Soc. 2010 Aug;69(3):400-6. DOI: $10.1017 / \mathrm{S} 0029665110001928$

33. Kasapis C, Thompson PD. The effects of physical activity on serum C-reactive protein and inflammatory markers: a systematic review. J Am Coll Cardiol. 2005 May 17;45(10):1563-9. DOI: 10.1016/j.jacc.2004.12.077

34. Febbraio MA, Pedersen BK. Muscle-derived interleukin-6: mechanisms for activation and possible biological roles. FASEB J. 2002 Sep;16(11):1335-47. DOI: 10.1096/fj.01-0876rev

35. Randers MB, Nielsen JJ, Bangsbo J, Krustrup P. Physiological response and activity profile in recreational small-sided football: No effect of the number of players. Scand J Med Sci Sports. 2014 Aug;24:130-7. DOI: $10.1111 / \mathrm{sms} .12232$

36. Nédélec M, McCall A, Carling C, Legall F, Berthoin S, Dupont G. Recovery in soccer. Sports Med. 2012 Dec 1;42(12):997-1015. DOI: 10.1007/BF03262308

37. Bais R, Edwards JB. Creatine kinase. Crit Rev Clin Lab Sci. 1982 Jan 1;16(4):291-335. DOI: $10.3109 / 10408368209107030$

38. Karamizrak SO, Ergen E, Töre IR, Akgün N. Changes in serum creatine kinase, lactate dehydrogenase and aldolase activities following supramaximal exercise in athletes. J Sports Med Phys Fitness 1994 Jun;34(2):1416.

39. Clarkson PM, Tremblay IS. Exercise-induced muscle damage, repair, and adaptation in humans. J Appl Physiol. 1988 Jul 1;65(1):1-6. DOI: 10.1152/jappl.1988.65.1.1

40. Clarkson PM, Dedrick ME. Exercise-induced muscle damage, repair, and adaptation in old and young subjects. J Gerontol. 1988 Jul 1;43(4):M91-6. DOI: 10.1093/geronj/43.4.M91 